\title{
Iron Abundances in AGB Stars and M Supergiant Stars at the Galactic Center
}

\author{
S. V. Ramírez ${ }^{1}$, K. Sellgren, D. M. Terndrup \\ The Ohio State University, Columbus OH 43210, USA
}

J. S. Carr

Naval Research Laboratory, Washington, DC 20375, USA

S. Balachandran

University of Maryland, College Park, MD 20742, USA

R. D. Blum

CTIO, La Serena, Chile

\begin{abstract}
We are measuring Fe abundances of cool, luminous stars within $30 \mathrm{pc}$ of the center of the Milky Way. Our sample contains both AGB stars and M supergiants. Low-resolution $(\lambda / \Delta \lambda=500) H$ and $K$ band spectra are used to estimate temperatures and gravities. Stellar Fe abundances are determined from high-resolution $(\lambda / \Delta \lambda=40000) K$ band spectra obtained on the IRTF using CSHELL. We find that Fe abundances of stars in the Galactic Center are consistent with the solar Fe abundance.
\end{abstract}

\section{Introduction}

We have measured iron abundances of cool luminous stars located within $30 \mathrm{pc}$ of Galactic Center (GC), using $K$-band $(2.2 \mu \mathrm{m})$ high-resolution spectra. Infrared techniques are required because of the large optical extinction towards the GC ( $A_{V} \sim 30$ mag.). Our sample includes both AGB stars and M supergiant stars.

Chemical abundances in the disks of spiral galaxies are observed to reach their highest values at the center (see Pagel \& Edmunds 1981 and Shields 1990). H II regions in the Milky Way also show a radial metallicity gradient (Peimbert et al. 1978; Hawley 1978; Shaver et al. 1983; Pipher et al. 1984; Simpson \& Rubin 1990; Simpson et al. 1995; Afflerbach et al. 1997). Chemical evolution models strive to explain these gradients by considering the relative star formation and gas infall/outflow rates, the star formation history, and the metal abundance of the gas compared to the stars (see Audouze \& Tinsley 1976 and Rana 1991). The gas metal abundance of the GC varies between 1 and 5 times solar depending

\footnotetext{
${ }^{1}$ US Gemini Fellow.
} 
on the element (Aitken et al. 1974, 1976; Willner et al. 1979; Lacy et al. 1980; Lester et al. 1981; Lester et al. 1987; Rubin et al. 1988; Shields \& Ferland 1994; Simpson et al. 1995; Afflerbach et al. 1997), but Fe has not been measured in the gas phase. Our measurements of $[\mathrm{Fe} / \mathrm{H}]$ are the first stellar abundance determinations in the GC.

\section{Observations}

We have observed cool luminous stars in the GC using the CSHELL spectrograph at the NASA Infrared Telescope Facility, a 3-m telescope located at Mauna Kea Observatory in Hawaii. The $K$-band spectra taken with this instrument have a spectral resolution of $\lambda / \Delta \lambda=40000$ or $7 \mathrm{~km} \mathrm{~s}^{-1}$. We have chosen iron lines which are free of molecular contamination, especially $\mathrm{CN}$ in $\mathrm{M}$ supergiants and water in $\mathrm{M}$ giant and AGB stars.

We have observed all cool stars that have low water absorption and that are brighter than $K=9$, which is the limit set by the sensitivity of CSHELL. Our sample consists of seven M supergiants and three AGB stars. Nine of these stars, including the three AGB stars, are located in the central cluster within $2.5 \mathrm{pc}$ of the GC. The remaining M supergiant belongs to the Quintuplet cluster, located $3 \mathrm{C}$ pc away from the central cluster.

\section{Abundance analysis}

For our abundance analysis we are using model atmospheres for late type giants and supergiants from Kurucz (1993) and Plez (1992), with the spectral synthesis program MOOG (Sneden 1973). We have found a good agreement between abundances derived from both sets of models in the range of stellar effective temperature $\left(T_{\text {eff }}\right)$ and surface gravity $(\log g)$ where they overlap. Our abundance analysis requires as input parameters: $T_{\text {eff }}, \log g$, the microturbulent velocity of the star $(\xi)$, and the oscillator strengths of the lines we are studying. We have verified our analysis technique for the GC stars by observing and analyzing cool luminous stars of known abundances (Smith \& Lambert 1985, 1986, 1990; Luck \& Bond 1989; McWilliam 1990).

Effective temperature: For all our GC stars, we use the $\mathrm{CO}$ and $\mathrm{H}_{2} \mathrm{O}$ absorption observed in the $H$ and $K$ bands at low resolution $(\lambda / \Delta \lambda=500)$ to get $\mathrm{T}_{\text {eff }}$. The CO strength increases with decreasing $\mathrm{T}_{\text {eff }}$ and decreasing $\log \mathrm{g}$. The $\mathrm{H}_{2} \mathrm{O}$ strength has the same $\mathrm{T}_{\text {eff }}$ dependence but opposite surface gravity dependence. These two features together provide 2-dimensional spectral classification (Kleinmann \& Hall 1986). Once we establish the luminosity class of each star, we use the $\mathrm{CO}$ strength to get $\mathrm{T}_{\text {eff }}$. In addition, we can use lines of $\mathrm{CO}$ present in $H$-band $(1.6 \mu \mathrm{m})$ high resolution spectra to get $\mathrm{T}_{\text {eff }}$. Lines with different lower excitation potentials give the same carbon abundance when $\mathrm{T}_{\text {eff }}$ is correct. We have observed these CO lines only in IRS 7, which is the brightest star in the GC. Both methods give the same value of $T_{\text {eff }}$ for IRS 7 .

Surface gravity: We derive the bolometric magnitude $\left(\mathrm{M}_{\mathrm{bol}}\right)$ of the GC stars using the observed $K$ magnitude, extinction, distance, and $\mathrm{T}_{\text {eff }}$. We locate the 
GC stars in the HR diagram, using $\mathrm{T}_{\text {eff }}$ and $\mathrm{M}_{\text {bol }}$. We estimate their masses by comparing their location in the HR diagram with evolutionary tracks for stars of different masses (Schaller et al. 1992; Schaerer et al. 1993a, b; Charbonnel et al. 1996). We compute the surface gravity using the estimated mass, luminosity and $\mathrm{T}_{\text {eff. }}$.

Microturbulent velocity: For all our GC stars, we estimate $\xi$ from $\log \mathrm{g}$ (McWilliam et al. 1995). In addition, we can use lines of CO present in the $H$-band to get $\xi$ spectroscopically. Lines of different equivalent widths give the same carbon abundance when $\xi$ is correct. We have observed these CO lines only in IRS 7. These two techniques agree for IRS 7 .

Oscillator strengths: We have derived empirical oscillator strengths for our abundance analysis. We use the spectrum of $\alpha$ Boo (Wallace \& Hinkle 1996), the abundance measurements of Peterson et al. (1993) for $\alpha$ Boo, and the synthetic spectrum generated by MOOG to derive gf-values.

We have studied how errors in $\mathrm{T}_{\text {eff }}$ and $\log \mathrm{g}$ would affect our Fe abundance determination. The Fe abundance is quite insensitive to errors in $\log \mathrm{g}$, since a typical error of \pm 0.2 in $\log g$ corresponds to an error in $[\mathrm{Fe} / \mathrm{H}]$ of \pm 0.05 dex. The Fe abundance is more sensitive to errors in $\mathrm{T}_{\text {eff }}$, since a typical error of \pm $200 \mathrm{~K}$ represents an error in $[\mathrm{Fe} / \mathrm{H}]$ of $\pm 0.1 \mathrm{dex}$.

\section{Results}

In the Table we present results for our sample of 10 stars within $30 \mathrm{pc}$ of the GC. Note that IRS 7 is the brightest star in the GC, and VR 5-7 is the star located at the Quintuplet cluster.

A statistical analysis gives us an unweighted mean of $[\mathrm{Fe} / \mathrm{H}]=+0.03$ and a mean weighted by the errors of $[\mathrm{Fe} / \mathrm{H}]=+0.13$. The estimate of the dispersion $(\sigma)$ is 0.20 dex. This is not significantly different from the error in $[\mathrm{Fe} / \mathrm{H}]$ for each star, given in the Table.

\begin{tabular}{lcc}
\hline Star & \# lines & {$[\mathrm{Fe} / \mathrm{H}]$} \\
\hline IRS 7 & 4 & $+0.08 \pm 0.18$ \\
IRS 11 & 3 & $+0.15 \pm 0.20$ \\
IRS 19 & 3 & $+0.30 \pm 0.09$ \\
IRS 22 & 4 & $+0.14 \pm 0.23$ \\
RCS 72 & 2 & $-0.16 \pm 0.34$ \\
RCS 114 & 2 & $-0.35 \pm 0.27$ \\
RCS 124 & 2 & $+0.19 \pm 0.09$ \\
RCS 129 & 2 & $+0.05 \pm 0.23$ \\
RCS 140 & 2 & $+0.09 \pm 0.43$ \\
VR 5-7 & 2 & $-0.18 \pm 0.13$ \\
\hline
\end{tabular}




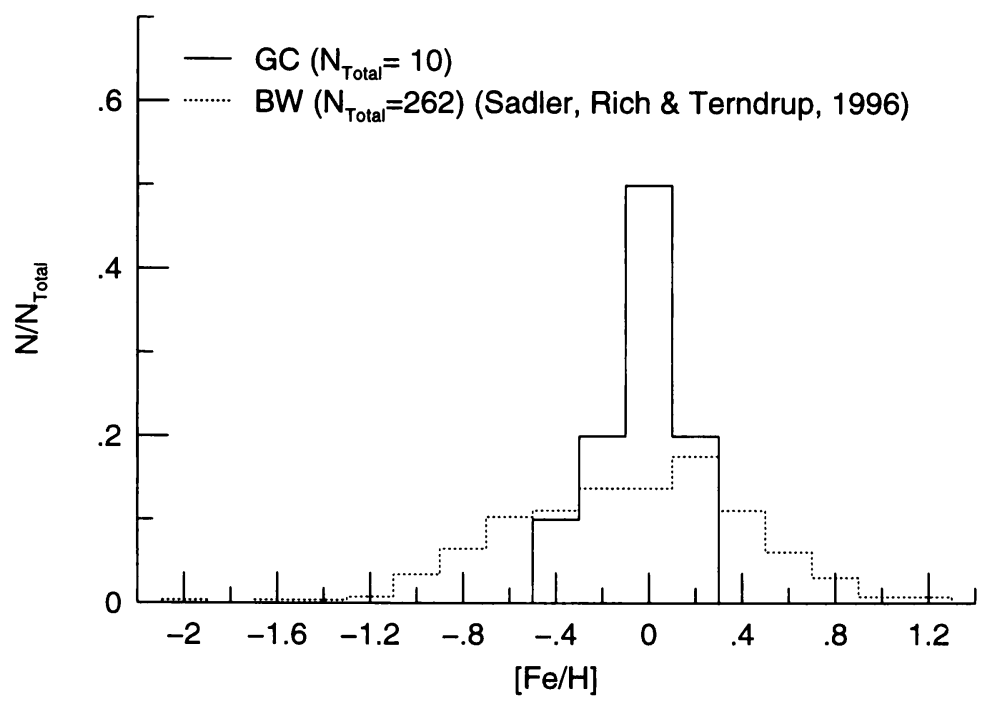

Figure 1. Fractional Fe abundance distribution of the GC compared with that of Baade's Window $\left(l, b=1^{\circ},-4^{\circ}\right)$ in the Galactic Bulge.

\section{Discussion}

In Fig. 1 we compare our $\mathrm{Fe}$ abundance distribution in the $\mathrm{GC}$ with the $\mathrm{Fe}$ abundance distribution of Baade's window in the Bulge (Sadler et al. 1996). Sadler et al. (1996) resolve the abundance spread of the Bulge distribution. We have not detected a $\mathrm{GC}[\mathrm{Fe} / \mathrm{H}]$ distribution that is wider than the errors, in spite of having similar errors per star as Sadler et al. (1996). Thus, the dispersion of the GC distribution is significantly narrower than that of the Bulge $[\mathrm{Fe} / \mathrm{H}]$ distribution.

In Fig. 2 we compare our GC Fe abundance distribution with the Fe abundance distribution of supergiants in the solar neighborhood (Luck \& Bond 1989). We see that the GC abundance distribution is consistent with the abundance distribution of supergiants in the solar neighborhood.

We find that the average GC stellar Fe abundance is near solar. This result differs from the gas abundances in the GC, which vary between 1 and 5 times solar, depending on the element. In the future, we will measure stellar abundances of $\alpha$-elements $(\mathrm{Ca}, \mathrm{Si}, \mathrm{Ti})$ to address the issue of selective enrichment in the GC.

\section{Conclusions}

- Galactic Center Fe abundances are consistent with the solar value.

- Galactic Center Fe abundance distribution is consistent with the Fe abundance distribution of supergiants in the solar neighborhood. 


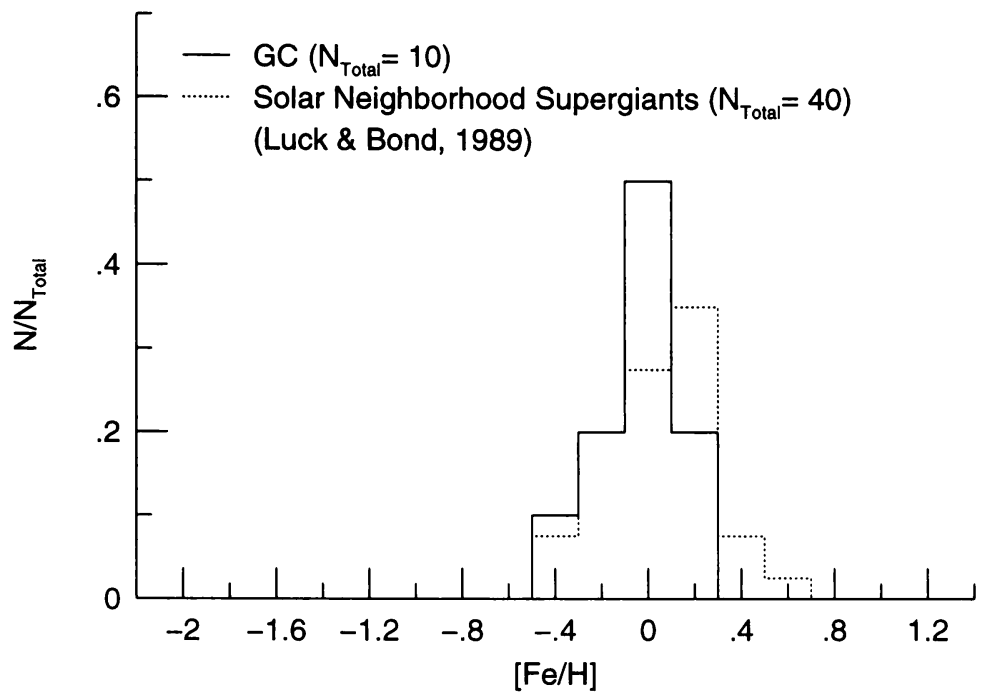

Figure 2. Fractional Fe abundance distribution of the GC compared with that of supergiants in the solar neighborhood.

- VR 5-7 (located 30 pc away from the center of the Galaxy) is not different in $[\mathrm{Fe} / \mathrm{H}]$ from stars located in the central cluster.

Acknowledgments. Support for this work was provided by the National Science Foundation through the grant \# GF-1003-97 from the Association of Universities for Research in Astronomy, Inc., under NSF cooperative agreement AST-8947990 and from Fundación Andes under project C-12984, and from NSF grant AST-9619230.

\section{References}

Afflerbach A., Churchwell E., Werner M.W., 1997, ApJ 478, 190

Aitken D.K., Jones B., Penman J.M., 1974, MNRAS 169, 35P

Aitken D.K., Griffiths J., Jones B., Penman J.M., 1976, MNRAS 174, 41P

Audouze J., Tinsley B.M., 1976, ARA\&A 14, 43

Charbonnel C., Meynet G., Maeder A., Schaerer D., 1996, A\&AS 115, 339

Hawley S.A., 1978, ApJ 224, 417

Kleinmann S.G., Hall D.N.B., 1986, ApJS 62, 501

Kurucz R.L., 1993, Kurucz CD-ROM No. 13 "ATLAS9 Stellar Atmosphere Programs and the $2 \mathrm{~km} \mathrm{~s}^{-1}$ Grid", Smithsonian Astrophysical Observatory, Cambridge

Lacy H.J., Townes C.H., Geballe T.R., Hollenbach D.J., 1980, ApJ 241, 132

Lester D.F., Bregman J.D., Witteborn F.C., Rank D.M., Dinerstein H.L., 1981, ApJ 248, 524 
Lester D.F., Dinerstein H.L., Werner M.W., Watson D.M., Genzel R., Storey J.W.V., 1987, ApJ 320, 573

Luck R.E., Bond H.E., 1989, ApJS 71, 559

McWilliam A., 1990, ApJS 74, 1075

McWilliam A., Preston G.W., Sneden C., Searle L., 1995, AJ 109, 2757

Pagel B.E.J., Edmunds M.G., 1981, ARA\&A 19, 77

Peimbert M., Torres-Peimbert S., Rayo J.F., 1978, ApJ 220, 516

Peterson R.C., Dalle Ore C.M., Kurucz R.L., 1993, ApJ 404, 333

Pipher J.L., Helfer H.L., Herter T., Briotta D.A., Houck J.R., Willner S.P., Jones, B., 1984, ApJ 285, 174

Plez B., 1992, A\&AS 94, 527

Rana N.C., 1991, ARA\&A 29, 129

Rubin R.H., Simpson J.P., Erickson E.F., Haas M.R., 1988, ApJ 327, 377

Sadler E.M., Rich R.M., Terndrup D.M., 1996, AJ 112, 171

Schaerer D., Meynet G., Maeder A., 1993a, A\&AS 98, 523

Schaerer D., Charbonnel C., Meynet, G., Maeder A., Schaller G., 1993b, A\&AS 102,339

Schaller G., Schaerer D., Meynet G., Maeder A., 1992, A\&AS 96, 269

Shaver P.A., McGee R.X., Newton L.M., Danks A.C., Pottasch S.R., 1983, MNRAS 204, 53

Shields G.A., 1990, ARA\&A 28525

Shields J.C., Ferland G.J., 1994, ApJ 430, 236

Simpson J.P., Rubin R.H., 1990, ApJ 354, 165

Simpson J.P., Colgan S.W.J., Rubin R.H., Erickson E.F., Haas M.R., 1995, ApJ 444, 721

Smith V.V., Lambert D.L., 1985, ApJ 294, 326

Smith V.V., Lambert D.L., 1986, ApJ 311, 843

Smith V.V., Lambert D.L., 1990, ApJS 72, 387

Sneden C., 1973, Ph.D. thesis, University of Texas

Wallace L., Hinkle K., 1996, ApJS 107, 312

Willner S.P., Russell R.W., Puetter R.C., Soifer B.T., Harvey P.M., 1979, ApJ 229, L65 